\title{
Vorsicht mit dem Begriff Lebensqualität!
}

«Meine Lebensqualität ist besser als vor der Krankheit, trotz meiner schweren Behinderungen.» [1]

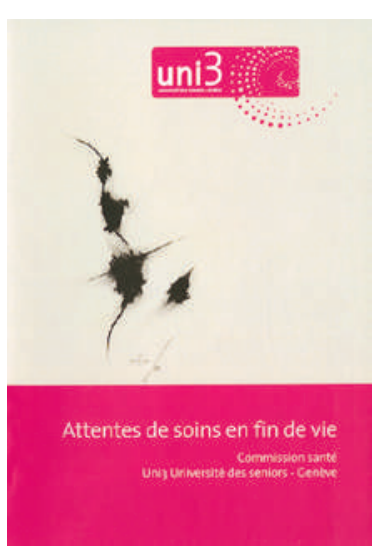

1 Aussage eines 48-jährigen Patienten mit amyotropher Lateralsklerose im fortgeschrittenen Stadium. In: Borasio GD. Mourir. Presses polytechniques et universitaires romandes; 2014. S. 78.

2 Commission Santé 2013: Attentes de soins en fin de vie. Université des séniors. Genf Download des Textes unter: www.unige.ch/uni3/ Commission/Publications. html

3 Bergner M. Quality of life, health status, and clinical research. Med Care. 1989; 27 (3 Suppl.):148-56.

4 Testa AM, Simonson DC. Assessment of quality-of-life outcomes. N Engl J Med. 1996; 334:835-40.

5 Oppikofer S. Informationsblatt Lebensqualität von Menschen mit einer Demenzerkrankung. Universität Zürich; 2013.

6 Arlt S, Hornung J, Eichenlaub M, Jahn H, Bullinger M, Petersen C. The patient with dementia, the caregiver and the doctor: cognition, depression and quality of life from three perspectives. Int J Geriatr Psychiatry. 2008;23(6):604-10.

hans.stalder[at]saez.ch
Arzt: «Wir tun alles, was in unserer Macht steht, denn sie hat eine gute Lebensqualität, sie ist jung - deshalb müssen wir alles tun, um sie zu heilen.»

Patientin: "Ich habe es Ihnen bereits gesagt: nur das absolut Notwendige.»

Arzt: «Diese Person hat noch eine angemessene Lebensqualität und nur wenige gravierende Probleme, abgesehen von der Tatsache, dass sie Hemiplegikerin ist; und sie wird von ihrem Mann sehr gut betreut.»

Patientin: «Ich habe Angst vor dem Leben, nicht aber vor dem Tod ... Dann ist man alle Probleme los (lacht).»

Arzt: «Da sie jung ist, eine gute Lebensqualität hat, ein gutes Umfeld und ein intaktes soziales Netz, werde ich in ihrem Fall alles in meiner Macht Stehende tun; ich würde sie auch ins Spital einweisen und, in ihrem konkreten Fall, falls nötig auf die Intensivpflege. Es handelt sich um jemanden [...], der noch voll urteilsfähig ist, ohne jegliche Anzeichen von geistigem Verfall [...] In diesem konkreten Fall würde ich aggressiv vorgehen.»

Patientin: "Lassen Sie mich in diesem Moment mein Leben beenden.»

Diese Antworten sind einer qualitativen Studie der Gesundheitskommission der Senioren-Universität Genf entnommen [2]. Den Bewohnern eines Altersund Pflegeheims und ihren Pflegekräften, Angehörigen und Ärzten wurden die folgenden Fragen gestellt: Sollte bei Ihnen plötzlich eine schwere Lungenentzündung auftreten, was wünschen Sie sich: ins Spital eingewiesen zu werden oder im Pflegeheim zu bleiben? Alle zur Verfügung stehenden therapeutischen Massnahmen oder nur palliative Medizin? Von den zwölf Insassen wollte nur eine hospitalisiert werden, während sieben von zwölf Ärzten sowie sieben Pflegepersonen und sechs Angehörige für die Hospitalisation optierten. Und nur zwei Insassen, aber alle Ärzte entschieden sich für den Einsatz aller verfügbaren Massnahmen. Nur bei einer gleichzeitig bestehenden Demenz und dem Auftreten einer akuten Pneumonie würden die meisten Ärzte auf eine Hospitalisation verzichten. Aber immer noch ein Drittel der Ärzte würde alle verfügbaren Behandlungen verordnen.

In ihren Antworten rechtfertigten die Ärzte ihre Entscheidung sehr häufig mit dem Begriff «Lebensqualität» (26-mal). Nur zwei Pflegekräfte und eine Bezugsperson verwendeten diesen Begriff und kein einziger der Heimbewohner ...

Der Begriff «Lebensqualität» ist nur schwer greifbar. Der WHO (1994) zufolge ist Lebensqualität die «subjektive Wahrnehmung einer Person über ihre Stellung im Leben in Relation zur Kultur und den Wertsystemen, in denen sie lebt, und in Bezug auf ihre Ziele, Erwartungen, Standards und Anliegen. Es handelt sich um einen weitgefassten Begriff, der auf komplexe Art das physische Wohlbefinden einer Person, ihren psychischen Zustand, den Grad der Unabhängigkeit, ihre sozialen Beziehungen, ihren persönlichen Glauben und ihre Beziehung zu den Besonderheiten ihrer Umwelt umfasst.»

In der medizinischen Literatur stösst man häufig auf den Begriff der «gesundheitsbezogenen Lebensqualität» (health-associated quality of life [3]). Er ist sowohl multidimensional (in seinen physischen, psychischen und sozialen Dimensionen) als auch subjektiv. Es gibt eine Unzahl von Fragebögen, um die Einschätzung der Betroffenen selbst zu erheben, denn die Lebensqualität kann nicht von Aussenstehenden, und somit auch nicht von Gesundheitsfachpersonen bewertet werden. Auch ist klar, dass unterschiedliche Messinstrumente angezeigt sind, je nachdem ob es sich um eine onkologische Patientin [4] oder eine betagte oder gar demente Person handelt $[5,6]$.

Der Begriff Lebensqualität wird schliesslich auch von Ökonomen verwendet, die ihn als quantifizierbare Einheit definieren, mit der der Nutzen einer zumeist onkologischen - Behandlung gemessen wird. Dabei wird berücksichtigt, dass nicht nur die Überlebensdauer massgeblich ist, sondern die Qualität des Lebens während dieser Dauer (QUALY). Wie aber werden QUALY-Werte bei einem bestimmten Patienten gemessen, wenn es sich bei der Lebensqualität um eine fundamental subjektive Wahrnehmung handelt?

Fazit: Wir sollten den Begriff «Lebensqualität» mit Vorsicht und Bedacht verwenden. Lassen wir doch unsere Patienten selbst definieren, was sie darunter verstehen, bevor wir den Begriff gebrauchen. Die Arbeit der Gesundheitskommission zeigt, dass Ärzte, Pflegekräfte und Angehörige sich mindestens in jedem zweiten Fall täuschen.

Übrigens: Wussten Sie, dass Zürich in der Beurteilung der Lebensqualität an zweiter Stelle (nach Wien) steht? Und dass das Zürcher Stimmvolk zu 85\% gegen ein Verbot der Sterbehilfe gestimmt hat? Offenbar ist auch die Qualität des Sterbens Teil der Lebensqualität ...

Hans Stalder*

* Prof. Dr. med. Hans Stalder, Facharzt für Innere Medizin, Redaktionsmitglied, ist ehemaliger Direktor der Policlinique de Médecine und des Département de Médecine communautaire des Hôpitaux Universitaires de Genève. 